\title{
Correction to: Comprehensive Analysis and Optimization of Dynamic Vibration-Absorbing Structures for Electric Vehicles Driven by In-Wheel Motors
}

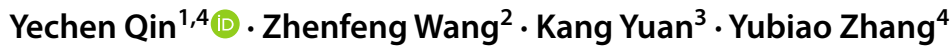 \\ Published online: 5 April 2020 \\ (c) The Author(s) 2020
}

\section{Correction to: Automotive Innovation (2019) 2:254-262 https://doi.org/10.1007/s42154-019-00079-9}

The original version of this article unfortunately contained a mistake. The last sentence of the abstract was incorrect. The corrected sentence is given below.
The original article can be found online at https://doi.org/10.1007/ s42154-019-00079-9.

\section{Yechen Qin}

qinyechenbit@gmail.com; qinyechen@bit.edu.cn

1 Beijing Institute of Technology, Beijing, China

2 China Automotive Engineering Research Institute Co Ltd, Tianjin, China

3 Chongqing University, Chongqing, China

4 University of Waterloo, Waterloo, Canada
"It can be concluded that either passenger comfort or motor lifespan can be improved up to $15 \%$ and $36 \%$ by properly changing the IWM suspension system parameters". 